\title{
DEVELOPMENT OF TOPOLOGY OPTIMIZED CABLE JOINT SYSTEM FOR OIL STORAGE TANKS
}

\author{
PARK, H[ong] S[eok] \& DAHAL, P[rakash]
}

\begin{abstract}
This paper presents the development procedure of a resilient cable joint that adaptively holds insulating layer outside the wall of oil storage tanks. The joint is developed and compared by a traditional design method and topology optimized method for evaluating the best method for implementation. The developed cable joint system produces an appropriate and reliable tension force that always firmly attaches the insulating layer to the tank wall regardless of the dimensional variation of the oil tank which is a function of seasonal ambient temperature and hydrostatic pressure. Systematic design procedure and finite element analysis were applied to calculate the complex variation of circumferential displacement of the tank wall. The developed flexible cable joint results in the better service duration and safer conditions for the cable and insulation system of the oil storage tank. Objective of the study is focused on minimizing volume of joint by maximizing stiffness. Lastly, it is concluded that topology optimized model maximizes overall stiffness resulting in better and innovative product design with enhanced performance.

Keywords: storage tank, flexible cable joint, mechanism design, topology optimization
\end{abstract}

\section{INTRODUCTION}

Large metallic cylindrical storage tank structures are widely used for storing petroleum products in a great volume [1].These tanks are placed in the outer environment so that environmental ambient temperature directly affects the oil stored inside the tank, making evaporative loss and hazardous conditions for fire or explosion. Therefore, they are provided with a suiTab. insulating material so that the products in the storage tanks are kept at the desired temperatures. Usually, the insulating layer is attached to the tank wall by the use of circumferential bands or steel cables extending around the outside diameter of the tank [2]. Because of the fact that the tank structure is exposed to the varying temperature gradient and the varying hydrostatic pressure during filling and draining process, the tank can be expanded or contracted compared to the installation status. When the tank expands, the bands can be broken or snapped off if the expansion is larger than their elastic elongation. In compression, the bands or cables that were experienced the undue stretching can lose their ability of holding the insulating layer to accommodate the contraction of the tank wall.

Consequently, the bond between the insulating layer and the tank wall is broken, or the insulating material is separated from the tank wall. As a result, the effect of the insulating layer on the safety of the oil tank is decreased, and the repair or maintenance process that needs the extra cost is required. Therefore, there is a necessicity to develop a joint system that can withstand the circumferential variation of oil tanks. However, a general design method of optimizing flexible joint system has not been developed yet. In addition, the failures of cable joint system due to improper design and complex of dimensional variation of the oil tank have not been investigated thoroughly. This study was carried out with the cooperation of SK Energy and KYE Company (Korea) in order to develop systematic design procedure and to propose the flexible resilient cable joint system that overcomes the above mentioned problem with lower cost, safety and higher reliability.

\section{DESIGN PROCEDURE OF NEW JOINT}

\subsection{Design specification}

The design specification was obtained from Company which includes tank size, wall thickness and insulation thickness given in Tab.1. The material used in tank and insulation are carbon steel foamglas respectively.

\begin{tabular}{|c|c|c|c|}
\hline $\begin{array}{c}\text { Tank } \\
\text { thickness }(\mathrm{m})\end{array}$ & $\begin{array}{c}\text { Tank } \\
\text { diameter(m) }\end{array}$ & $\begin{array}{c}\text { Tank } \\
\text { height }(\mathrm{m})\end{array}$ & $\begin{array}{c}\text { Insulation } \\
\text { thickness(m) }\end{array}$ \\
\hline 0.015 & 35 & 20 & 0.1 \\
\hline
\end{tabular}

Tab. 1.Dimensions of tank obtained from Manufacturer Company.

Design requirements are the set of terminologies which are used to develop the final approach of a system.They can be classified into physical, structural, functional, cost requirements. The design requirements of cable joint system are as follows:

- Cable joint must bear high strength and reliability to resist constant load exerted by the tank for a long time.

- Resistance to corrosion for a long time is necessary for joint because it is enclosed in the place between aluminium layer and insulation where the humidity and moisture can affect the material.

- System must be safe for the insulation during tank wall movement due to ambient temperature variation $\left(-15^{\circ} \mathrm{C}\right.$ at winter and $35^{\circ} \mathrm{C}$ at the summer).

- Cable assembled with joints must be permitted to expand and roll without any negative effect in insulation.

Therefore, to have the chance of new reliable and efficient cable joint being implemented, system must be long lasting, requiring no maintenance and designed by sequential design process is preferred. 


\subsection{Design procedure}

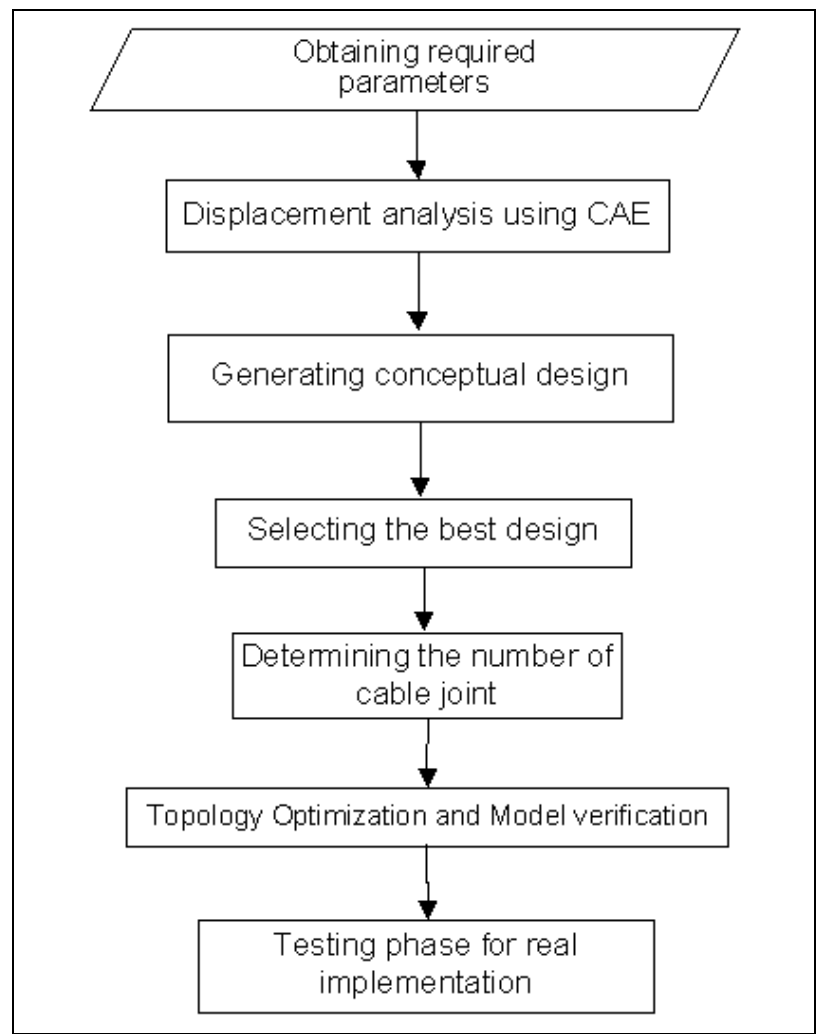

Fig.1. Schematic of systematic design process

The functional design of cable joint system starts with collection of necessary parameters including dimensions of tank, material used and cable specifications which are used in the existing tanks. Dimensions of tank are used to generate $\mathrm{CAD}$ model for displacement variation analysis.

Displacement analysis can be done from data obtained from hydrostatic loading and change of ambient temperature. Ambient temperature is taken from the highest and lowest temperature obtained throughout the year whereas hydrostatic loading is dependent on the level of oil in the tank. Obtained results from the analysis are used to calculate circumferential variation of tank wall and processed to determine the number of resilient joint to be used. Innovative cable joints are designed in terms of performance, cost and ease of manufacturability and best viable structure is selected. A part of the selected model is verified by FEA procedure and topology optimized structure is made for robust design and compared with the traditional design for verification.

\subsection{Displacement analysis}

This is the main step to develop the cable tension joint because main data necessary to develop the joint can be obtained from the result of analysis. Loading due to hydrostatic pressure and thermal conditions are perfectly applied in the walls of the storage tank using axisymmetric CAD model. Total hydrostatic pressure of $150093 \mathrm{~Pa}$ is applied on the walls of storage tank. Whereas variable thermal load of $-150 \mathrm{C}$ to $350 \mathrm{C}$ is applied according to the analysis done during winter and summer conditions.

Linear static analysis using FEA method is performed under displacement to investigate the expansion or contraction behavior in different regions at different nodes of the storage tank. During the displacement analysis, distributed force due to tightened cable in the outside circumference is also considered for the accuracy. The maximum spring force to hold the insulation is obtained at the place of maximum displacement of $282.40 \mathrm{~N} / \mathrm{m}$. Numbers of circumferential cables are taken from the manufacturer's data $1500 \mathrm{~mm}$ apart vertically as shown in Fig. 2 .

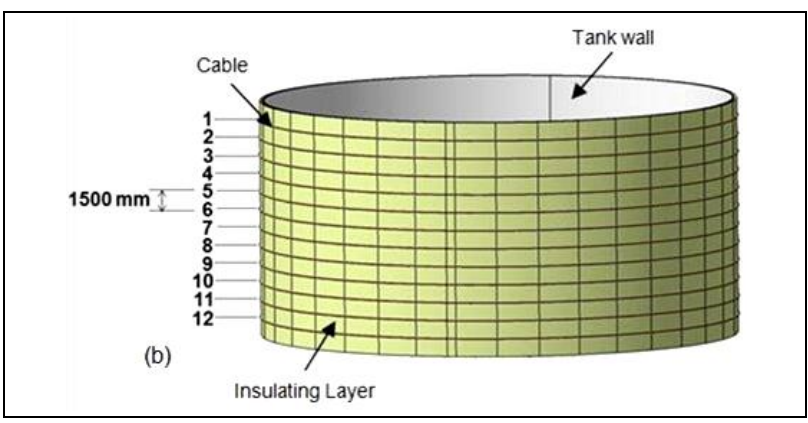

Fig.2. Position of cable with numbers shown in 3D model

To perform displacement analysis, there are two factors to be considered: 1) Climatic and 2) Hydrostatic. Climatic loading depends on ambient temperature which is determined from minimum and maximum temperature within a year. For hydrostatic loading, maximum capacity of oil is assumed to be 90 percent of total capacity to prevent overfilling with potential fire risk and oil spill [3].

After the analysis, obtained radial displacement results are used to calculate circumferential displacement, number of springs and joints to be used. The area of higher displacement needs more spring because each spring has a certain maximum working stroke. Size of cable, weight of insulation material, number of rings and outer panel is fixed in the present study because they are predetermined by experience of the manufacturer. Due to the variation of displacement in upper part and lower part of tank, number of resilient cable joints to be installed also varies circumferentially.

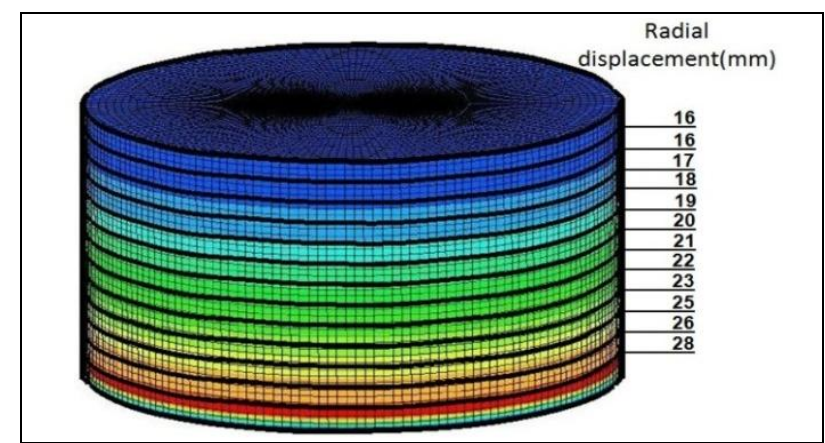

Fig.3. Analysis result of displacement

Fig. 3 shows the result of total deflection of tank wall at the critical conditions during winter (when the temperature is $-15^{\circ} \mathrm{C}$ ) and summer (when the temperature is $35^{\circ} \mathrm{C}$ ) by assuming that base temperature is $20^{\circ} \mathrm{C}$ during the installation. Uppermost cable no 1 has minimum displacement at the summer because of minimum hydrostatic force and maximum displacement at winter due to high ambient temperature. Overall 
displacement is the difference of displacement during winter from displacement during summer. Maximum circumferential displacement obtained is $178.94 \mathrm{~mm}$ which is used to determine the number of joints to be used on the upcoming section.

\subsection{Conceptual design}

The goal of concept generation is to thoroughly explore the space of the product concepts that may address the target specification. Concept generation includes a mixing of external search, creative problem solving and systematic exploration of the various solution fragments. [4] The result of this activity are three generative concepts, each typically represented by a concept model and working mechanism. Four concepts are generated via CAD model where each model satisfies the design requirement as shown in Fig.4.

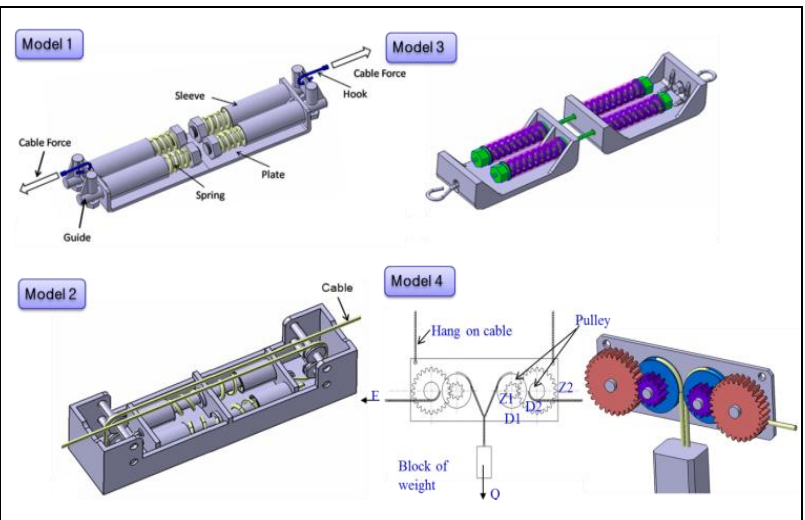

Fig.4. Conceptual design of concrete pipe making machines

In Model 1 and Model 3, hook is used for transmitting the force exerted by cable to spring. Spring placed between sleeve and guide is used for storing or releasing energy to pull the insulation. There are less moving parts and strong framework which increase durability in those models. Model 2 shows a system prepared by the arrangement of roller and joints.

The important function of roller in this system being, transmitting the force of one height to another height in an opposite direction, it also acts as an intermediate to release stored force of spring. Model 4 shows an extraordinary and distinctive system which gives copious profit of making constant force throughout the operational life. However, it is complex because huge blocks of weight are used to give constant tension force to the cable and the blocks of weight needs the big space which is not practicable.

\subsection{Concept selection}

Concept scoring technique is used to select best model from the generated designs. To reflect the result of best selected concept, relevant issues are considered and compared with each model to satisfy the design requirements. Rating of 1 to 5 is given according to relative performance by comparing with reference model whereas 1 to 9 is used to give weights for selection criteria. Each weight is given to individual selection criteria and focus on more refined comparisons with respect with each criterion determined by weighted sum of the ratings given by,

$$
S_{j}=\sum_{i=1}^{n} r_{i j} w_{i}
$$

\begin{tabular}{|c|c|c|c|c|c|c|c|c|}
\hline \multirow[t]{2}{*}{ Concepts } & \multicolumn{2}{|c|}{ Model 1} & \multicolumn{2}{|c|}{ Model2 } & \multicolumn{2}{|r|}{ Model 3} & \multicolumn{2}{|r|}{ Model 4} \\
\hline & Weight Rating & Weighted score $R$ & Rating V & \multicolumn{2}{|c|}{ ng Weighted score Rating } & \multicolumn{2}{|c|}{ Weighted score Rating } & Weighted score \\
\hline \multicolumn{9}{|l|}{ Selection criteria } \\
\hline No need of maintenance & 7 & 28 & 3 & 21 & 4 & 28 & 4 & 28 \\
\hline Durab| Strong framework & 9 & 36 & 2 & 18 & 4 & 36 & 4 & 36 \\
\hline Less parts & 3 & 9 & 1 & 3 & 4 & 12 & 3 & 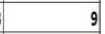 \\
\hline Light weight & 1 & 5 & 2 & 2 & 5 & 5 & 1 & 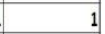 \\
\hline Safety No sharp edge & 3 & 9 & 3 & 9 & 2 & 6 & 3 & 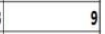 \\
\hline No moving parts on base & 3 & 15 & 5 & 15 & 2 & 6 & 3 & 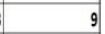 \\
\hline \multirow[t]{2}{*}{ Easy to manufacture } & 3 & 9 & 3 & 9 & 3 & 9 & 4 & 12 \\
\hline & Total rank & 111 & & 77 & & 102 & & 104 \\
\hline Continue? & & Yes & & No & & No & & No \\
\hline
\end{tabular}

Tab. 2. Concept scoring matrix

Where, $\mathrm{n}$ is the number of selection criteria, rij is the rating of concept $j$ for ith criterion and $w_{i}$ is the rating of the ith criteria. The overall performances of the alternative designs are ranked based on their total score. The relative strengths and weaknesses of alternative concepts can be identified by investigating the concept of scoring matrix which can be seen in Tab. 1. Weighted rating of each model is compared and best design is selected to proceed for continuation.

In the Tab. shown above, modell get the highest score comparing with the all model so it is implemented further for real use. However, the goal of the research is to compare the traditional design and topology optimized design in terms of stiffness and find out the best joint, therefore, for the selected joint structural topology optimization is done which is carried out in the next section.

\section{TOPOLOGY OPTIMIZATION}

Topology optimization is carried out to remove material from the selected model. Optimization process used in current research is followed by several design steps firstly, by making design domain of CAD model in CATIA. Next, Hypermesh was used to import model for finite element modeling where loads and constraints are applied. Furthermore, Hyperview and Hypergraph were used to display and plot the data for results interpretation. [5] Results of optimization runs performed with individual loads applied separately help to identify preferred load path with respect to the type of load applied. Finite element analysis is used in first stage before performing optimization for analyzying the initially generated design.

Optimization is performed by taking design space of joint from the traditional model. Optimization problem is expressed as :

\section{Minimize f(volume); Subject to $0 \leq \rho \leq 1$, Displacement $\leq 0.1 \mathrm{~mm}$, Stress $\leq 3.09 \mathrm{e} 7$.}

Finite element analysis is done to find out the stress and displacement by performing analysis on existing model. Stress and displacement used in optimization problem are taken from the analysis of initial model and are applied as constraints for optimization problem. The design space is created and has been meshed with solid tetrahedral elements. The boundary conditions are defined by fixing the bottom parts which are in connection with the tank. 


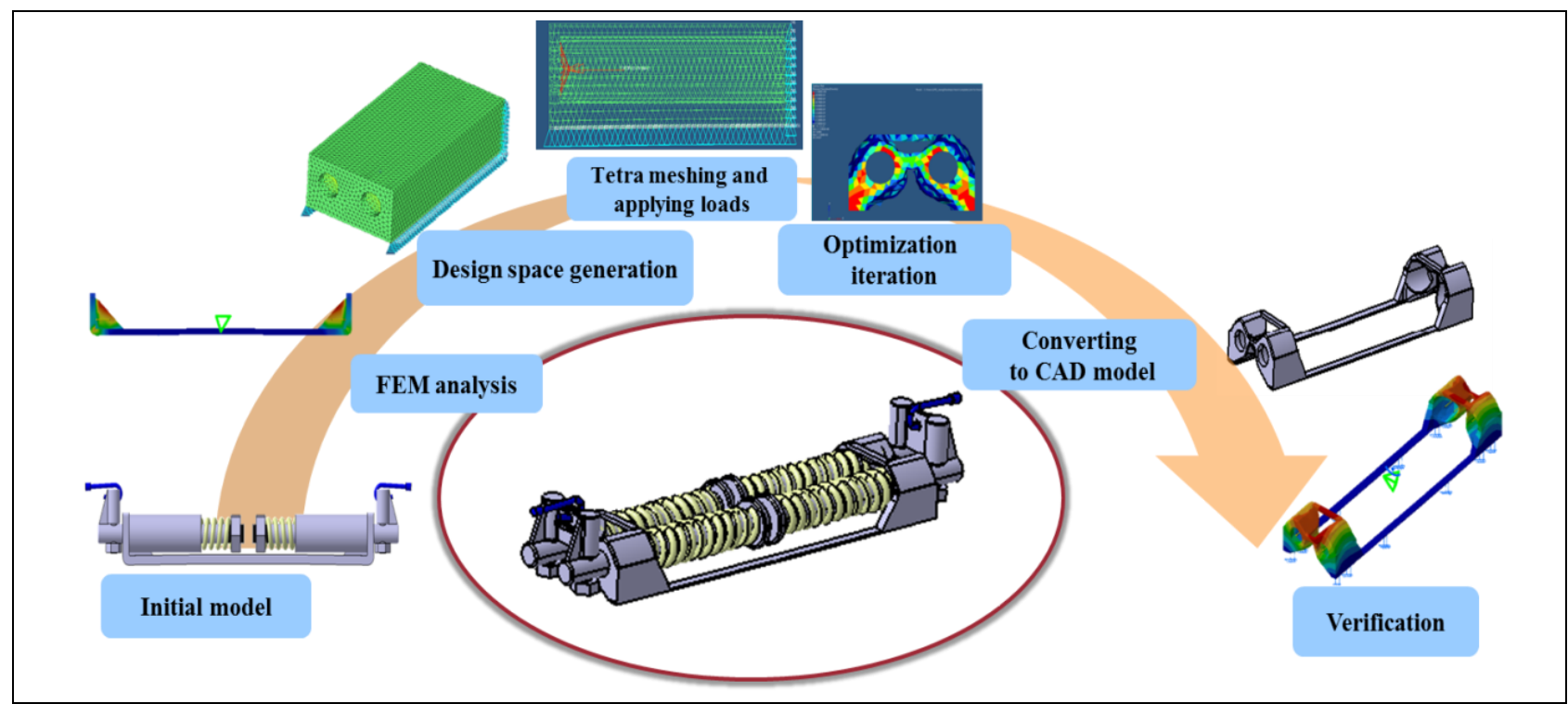

Fig.5. Selected Conceptual design followed by topology optimization process

The sleeve holes are directed to a force in horizontal direction produced by contraction of spring or expansion of oil storage tank. Design domain is created considering symmetry at the middle part so that calculation time of iteration is minimized. After the optimization has processed, model is adjusted for a series of steps until the convergence is reached. The resulting converged geometry proposal obtained from optimization process can be seen in Fig. 5 .

Although the topology results appear reasonable, the design is not ready to hand over to the machine shop for fabrication. The results of the topology studies are merely rough geometric proposals and some interpretation is required to create the final design. The optimized shape was created using CATIAV5 from the proposed design.Sketch was done over the generated rough model for creation of the CAD model and the interpretation of whole optimization process was done.

The design retains the features proposed by OptiStruct to get better and efficient performance within the minimization of weight. The generated model of the joint has been produced by reducing weight of $32 \%$ than initial design. The analysis results are compared between conventionally designed model and topology optimized model. The displacement results are reduced from $0.1 \mathrm{~mm}$ to $0.004 \mathrm{~mm}$ and stress level is reduced to $8.4 \mathrm{e} 6$ from $3.09 \mathrm{e} 7 \mathrm{~N} / \mathrm{m} 2$ during comparision between the conventional and optimized model. Therefore, optimized model is considered as better for the application for real use.

\section{CONCLUSION}

A systematic development of the cable joints mechanism for the insulating system in the oil tank was presented in order to prevent the failure of the insulating system caused by the variation of environmental temperature and the change of static hydraulic pressure.

Two different resilient cable joint were proposed and compared with the traditional method and topology optimization method, for which both of the joints enhance the durability and increase the performance of storage tanks because the potential maximum dimensional variation of the tank is analyzed elaborately. Due to the new optimized design concept and proper design process, optimized design was found to be lesser weight having more reliability and satisfied the requirement of the oil company who cooperates in this study.

Future works are focused to manufacture the optimized joint for testing the durability and reliability for real verification. Real testing is impossible in this case for large storage tanks, which the limitation for current study is. However, increasing the load magnitude and load cycle in order to reduce the testing time is also the appropriate test for the case of the developed cable joints in oil tank because they work in very low cycle load. It can be concluded that the cable joints fulfill the design requirement of the tank manufacturer company.

\section{ACKNOWLEDGEMENTS}

This work (Grants No.2011-0520) was supported by Business for Cooperative R\&D between Industry, Academy, and Research Institute funded Korea Small and Medium Business Administration in 2011.

\section{REFERENCES}

[1] Uwe Hornung, Helmut Saal, Buckling loads of tank shells with imperfections, International Journal of Non Linear Mechanics 37 (2002) 605-621

[2] *** (2012) Tufseam standard panel system.( website), http:// www.tufseam.com/applications.html, Accessed on: 2012-08-31

[3] Luis A. Godoy, and Eduardo M. Sosa. Deflections of ThinWalled Storage Tanks with Roof due to Localized Support Settlements. Department of Civil Engineering, University of Puerto Rico, Mayagüez Campus. Mayagüez 00681-9041, Puerto Rico

[4] Chang-Hee Cho and Kwon-Hee Kim, Product development with TRIZ: design evolution of deburring tools for intersect-ing holes, Journal of Mechanical Science and Technology 24 (2010) 169 173

[5] Altair Engineering Inc. Optistruct 10.0 help files, 2009 